\title{
Panorama de la energía renovable en Centroamérica
}

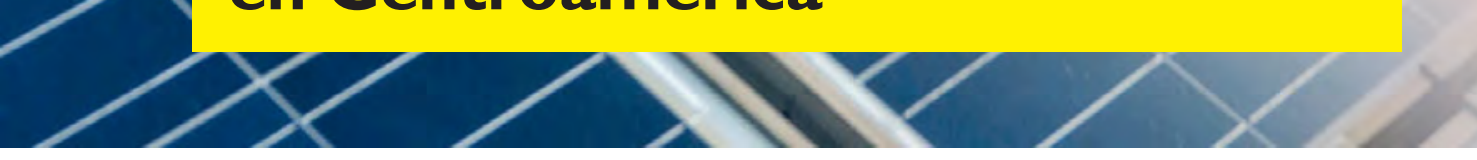

Por Ernest \& Young El Salvador S.A. de C.V.

\section{PANORAMA GENERAL REGIONAL}

Los países desarrollados basaron su estrategia de crecimiento en la sostenibilidad energética. Este concepto engloba la seguridad y la equidad energética junto a su sostenibilidad ambiental, la cual incluye el desarrollo de fuentes renovables y la disminución de las emisiones de carbono.

\section{:- KEY FACTS}

- De acuerdo con la CEPAL, para diciembre del 2015, la capacidad instalada en la región aumentó 9.2\% (1,602 $\mathrm{MW}$ ) con respecto el año pasado. Del total de la capacidad instalada en los países de la región (19.993 MW), el $54 \%$ corresponde a energías renovables.

- En el 2015 se generó energía eléctrica de distintas fuentes; los hidrocarburos y los combustibles fósiles representan el $47,6 \%$, la hidráulica el $36,3 \%$, la geotermia el $5,7 \%$, la eólica el $5,3 \%$, la solar un $0.9 \%$ y el biogás representa una pequeña fracción.

- La producción eléctrica en el 2015 creció un 4.7\% con respecto el 2014. La producción se encuentra distribuida de la siguiente manera: $0,5 \%$ en Belice, $6,5 \%$ en Nicaragua, $8,8 \%$ en El Salvador, $13,1 \%$ en Honduras, $15,1 \%$ en Panamá, $16 \%$ en Guatemala, $16,6 \%$ en Costa Rica y un 23,2\% en República Dominicana.
- En dicho año la energía eléctrica se generó de las siguientes fuentes: un $47,6 \%$ provino de energías fósiles e hidrocarburos, un 36,3\% de la energía hidráulica, un 5.7\% de la energía geotérmica, un 5,3\% de la energía eólica, un 4,2\% del bagazo de caña, un $0.9 \%$ de energía solar y una pequeña fracción proveniente del biogás.

- De acuerdo con el informe de la CEPAL, en el 2015 las ventas de electricidad al consumidor final regulado, en los países del SICA, ascendieron a 6,8 mil millones de dólares.

- Según Centralamericadata entre los meses de mayo del 2016 y 2017 , se presentaron 27 estudios de impacto ambiental para el desarrollo de energías renovables, cuya inversión podría ascender a $\$ 1.200$ millones. Dicha inversión se distribuyó de la siguiente manera: Panamá (\$877 millones), Costa Rica (\$254 millones), Guatemala (\$24 millones) y El Salvador (\$7 millones).

La información anterior resulta relevante porque muestra que a pesar de los esfuerzos de los gobiernos y de las empresas privadas en promover y desarrollar energías renovables, aún existen oportunidades para aumentar la participación de esta.

Este Sector Knowledge tiene como objetivo explorar el contexto de la energía renovable en la región. De manera especifica, contiene información sobre la capacidad instalada de la energía renovable, las cifras de inversión en dicho rubro, los principales proyectos en construcción y los principales retos a futuro. Aunado a lo anterior, EY posee una amplia gama de servicios con los cuales puede apoyar a las empresas públicas y privadas que deseen incursionar en el ámbito de la energía renovable. Finalmente, se describen cuales son las principales tendencias de las energías renovables a nivel mundial, las cuales impactarían nuestra región. 


\section{(2) GUATEMALA}

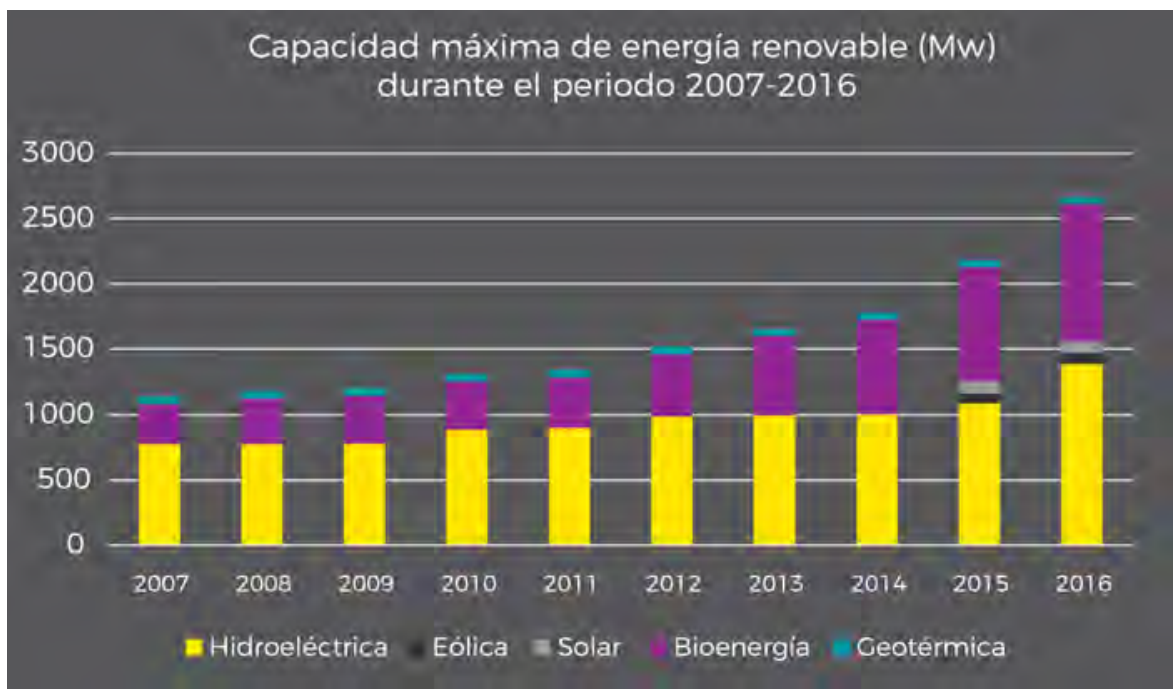

La matriz de energía renovables se encuentra altamente diversificada. La energía hidroeléctrica y la bioenergética son las más relevantes.

La energía eólica y solar comenzaron a crecer a partir del año 2015.

\section{PROYECTOS FUTUROS}

$\begin{array}{llll}\text { Nombre } & \text { Tipo } & \begin{array}{l}\text { Inversión (US\$ } \\ \text { millones) }\end{array} & \text { Generación (megavatios) } \\ \text { Parque Eólico Las Cumbres } & \text { Eólica } & \text { N.D } & 30 \\ \text { Proyecto Eólico Comapa } & \text { Eólica } & \text { N.D } & 57\end{array}$

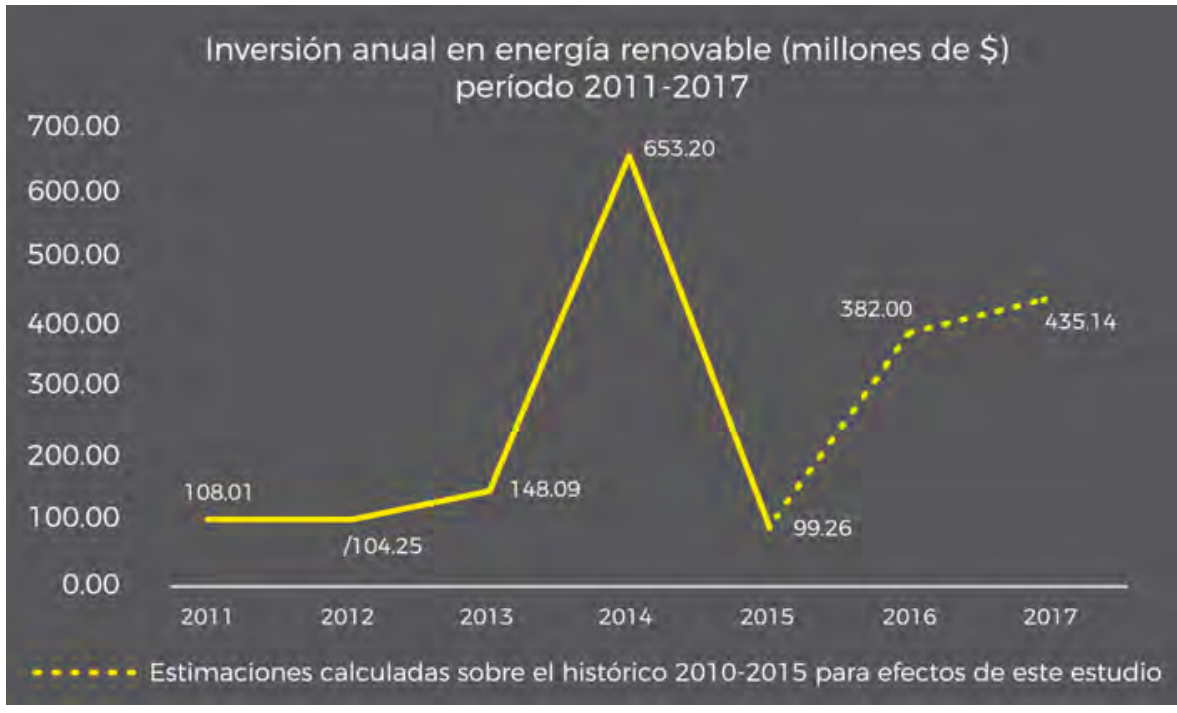

De acuerdo con el gráfico anterior, se puede observar que la inversión aumentó considerablemente en el 2014, sin embargo decreció sustancialmente en el 2015.

\section{$64 \%$}

\section{de generación de energía} limpia en 2016.

\section{Retos país:}

- Se deben promover incentivos para fomentar la inversión en energía renovables (Ejemplo incentivos fiscales y de inversión, subsidios, entre otros).

- Actualización de estudios sobre el potencial de los recursos renovables.

- Fortalecer seguridad jurídica. 


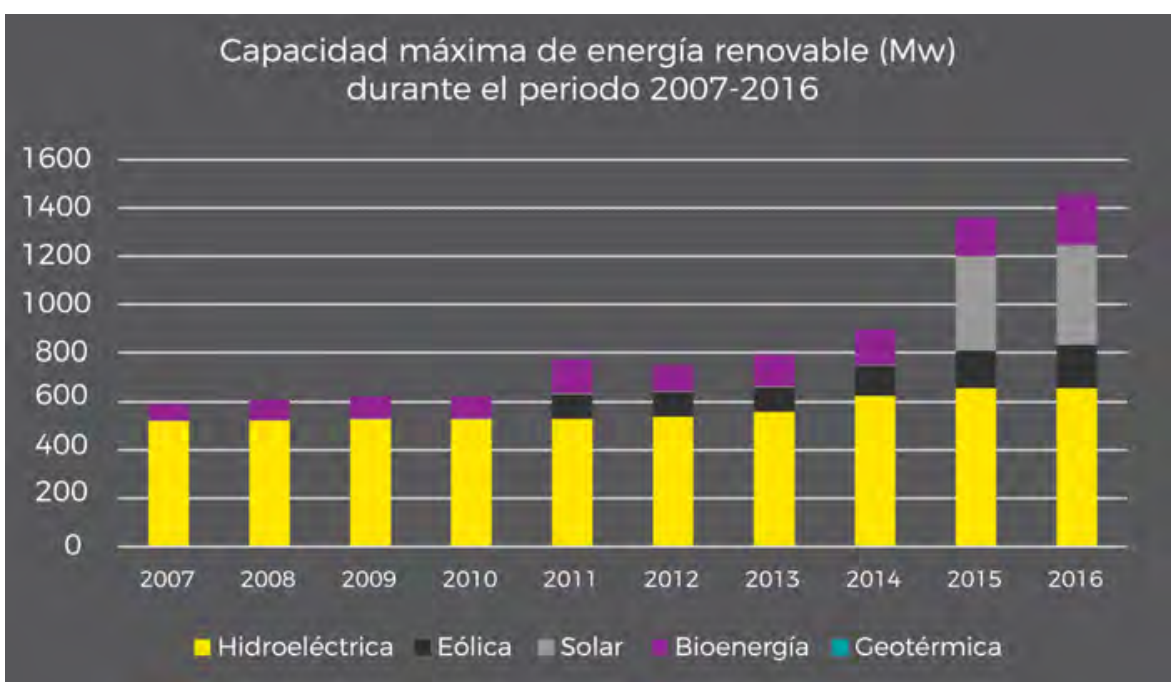

La energía hidroeléctrica es la más relevante, sin embargo desde el 2014 la energía solar ha crecido considerablemente.

\section{A Proyectos futuros}

\begin{tabular}{|llll|} 
Nombre & Tipo & $\begin{array}{l}\text { Inversión (US\$ } \\
\text { millones) }\end{array}$ & Generación (megavatios) \\
Patuca III & Hidroeléctrica & 347 & 104 \\
Llanitos y Jicatuyo & Hidroeléctrica & 800 & 300 \\
Proyecto Geotérmico Platanares & Geotérmica & 200 & 35 \\
\hline
\end{tabular}

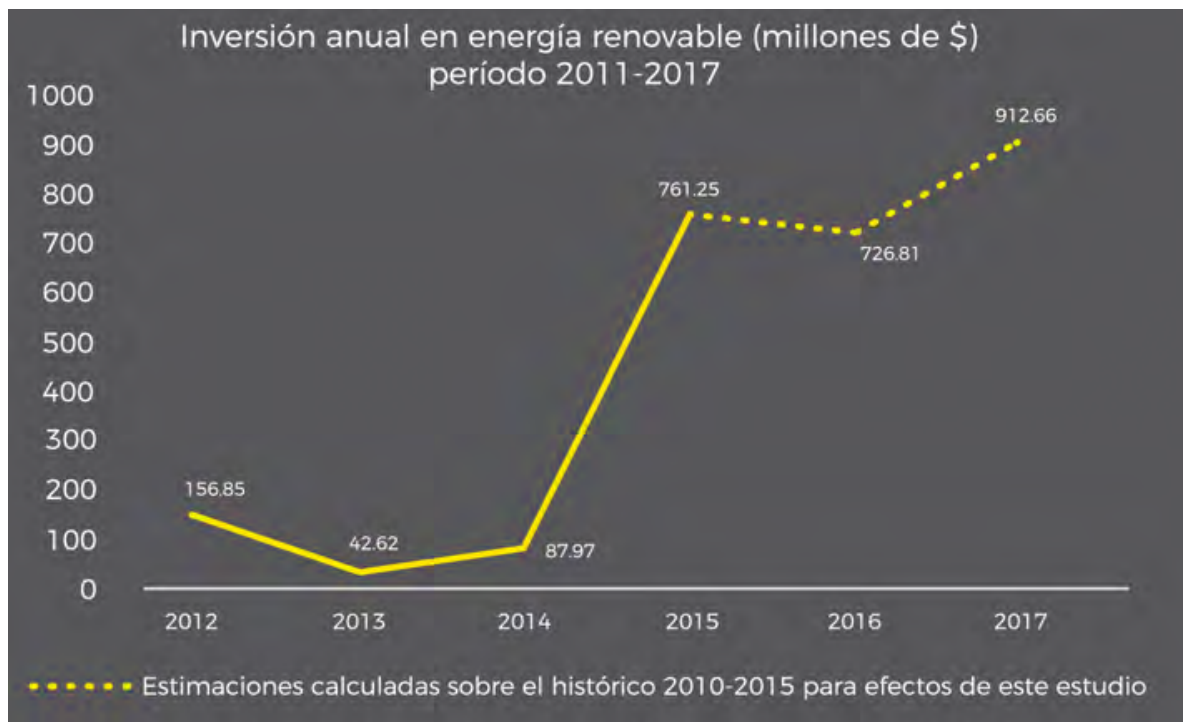

\section{$50.1 \%$}

\section{de generación de energía limpia en 2016.}

\section{Retos país:}

- Ejecución de estudios ambientales que sean inclusivos y que otorguen viabilidad de los proyectos en diferentes zonas del país.

- Búsqueda de fuentes de financiamiento para lograr mayor desarrollo.

La inversión disminuyó entre los años del 2012 - 2014, sin embargo aumentó considerablemente en el 2015. 


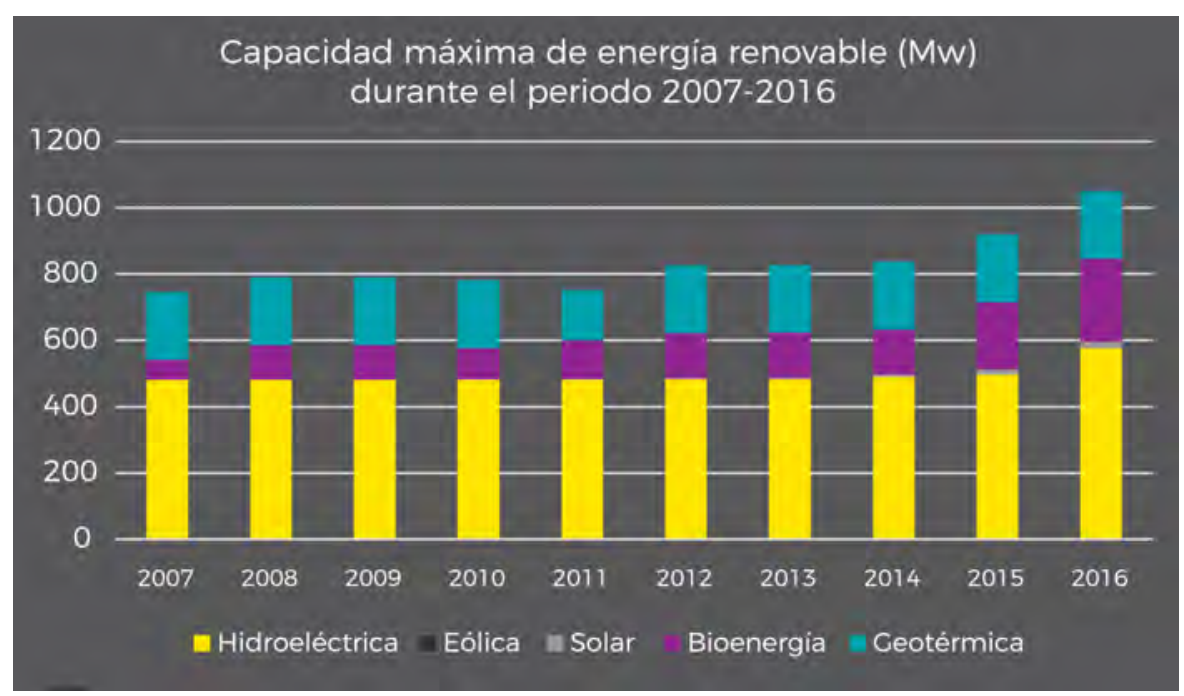

La generación hidroeléctrica es la más relevante, seguido de la bioenergía y la geotérmica.

\section{A PROYECTOS FUTUROS}

$\begin{array}{llll}\text { Nombre } & \text { Tipo } & \begin{array}{l}\text { Inversión (US\$ } \\ \text { millones) }\end{array} & \text { Generación (megavatios) } \\ \text { Parque Eólico El Cuajoyo } & \text { Eólica } & 110 & 50 \\ \text { Ozatlán } & \text { Solar } & 150 & 50 \\ \text { Ajacutla y Ateos } & \text { Solar } & \text { N.D } & 10 \\ \text { Bósforo } & \text { Solar } & 160 & 100\end{array}$

Inversión anual en energía renovable (millones de \$) período 2011-2017

Para 2013 se habían invertido $\$ 224.84$. Que en 2014 se convirtió en un monto nulo y se retomó en 2015 con una inversión de \$89.1.

\begin{tabular}{|ccccc|}
2011 & 2012 & 2013 & 2014 & 2015 \\
0 & 0 & 224.84 & 0 & 89.1 \\
\hline
\end{tabular}

Nota: En este caso no se agrega el gráfico ya que los datos existentes son escasos.

La inversión disminuyó entre los años del 2012 - 2014, sin embargo aumentó considerablemente en el 2015.

\section{de generación de energía limpia en 2016.}

\section{Retos país:}

- Diversificación de la matriz energética

- Mejorar de la seguridad jurídica

- Búsqueda de fuentes de financiamiento 


\section{(4) NICARAGUA}

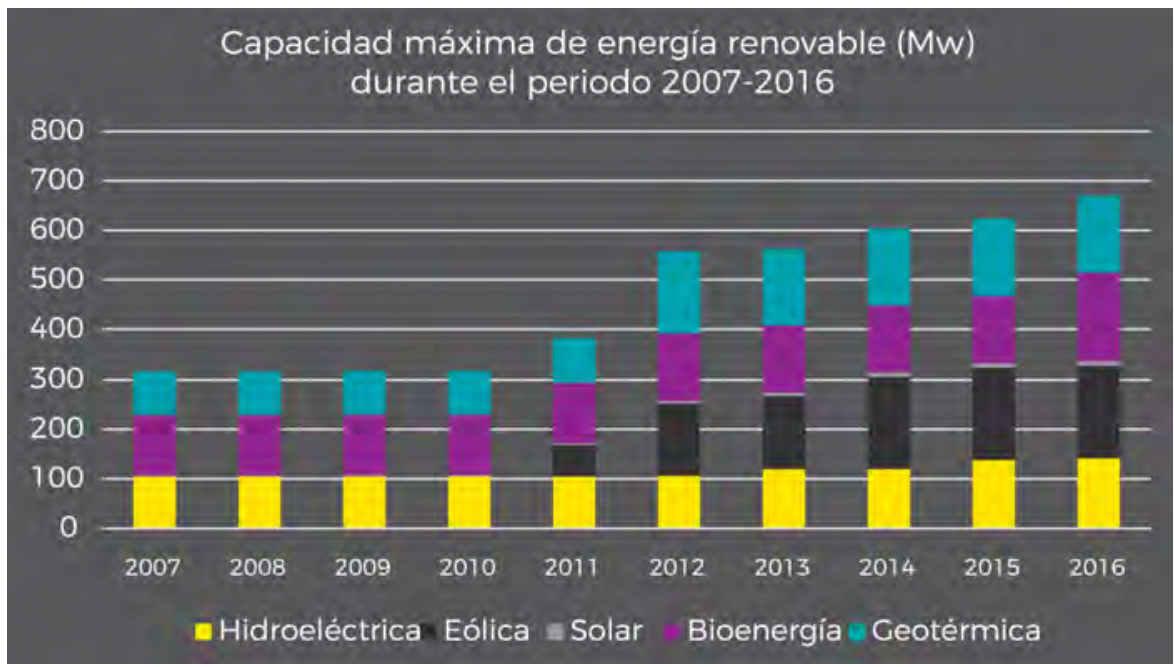

La matriz energética se considera diversificada. La energía más relevante es la eólica y la bioenergética.

\section{(1) PROYECTOS FUTUROS}

\begin{tabular}{llll}
\hline Nombre & Tipo & $\begin{array}{l}\text { Inversión (US\$ } \\
\text { millones) }\end{array}$ & Generación (megavatios) \\
Camilo Ortega II & Eólica & N.D & 40 \\
Puerto Sandino (expansión) & Solar & N.D & 100 \\
Ingenio Benjamin Zeledón & Biomasa & 90 & 35 \\
Ato Grande & Eólica & N.D & 23
\end{tabular}

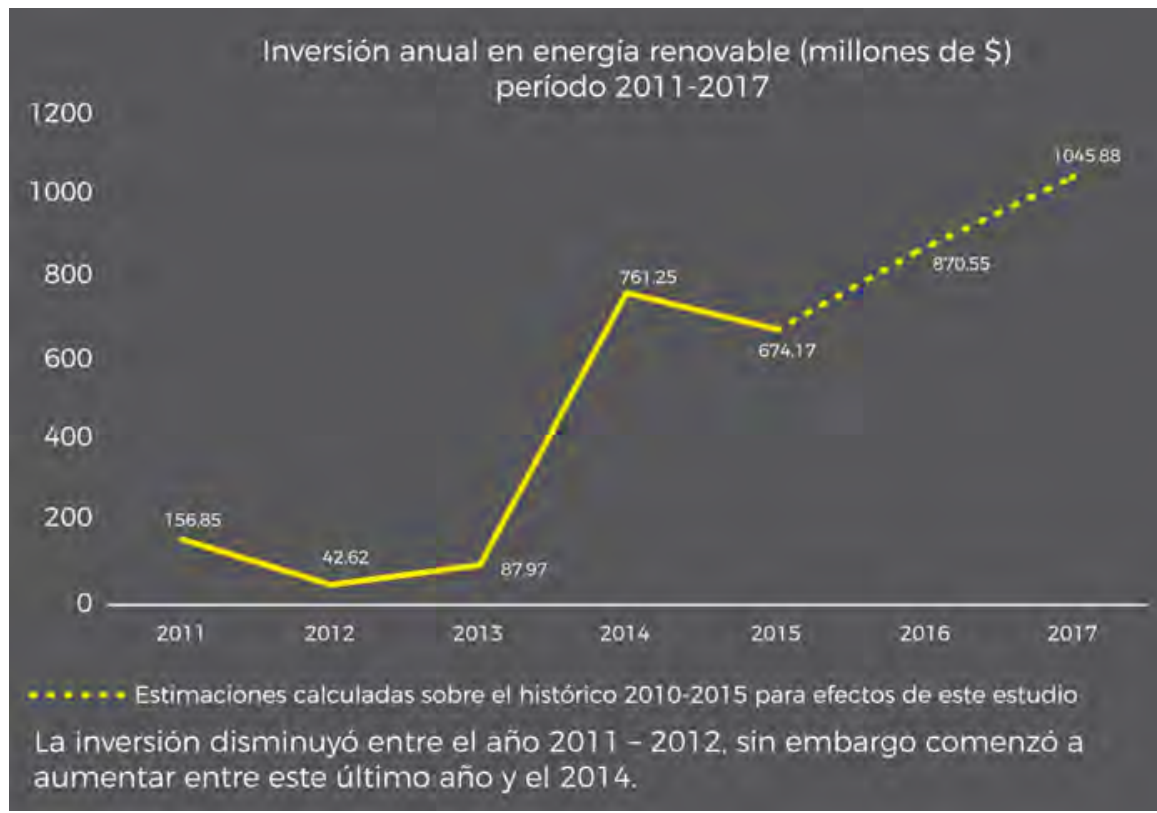

\section{de generación de energía limpia en 2016.}

\section{Retos país:}

- Mejorar la transparencia en la asignación de las concesiones junto al fortalecimiento de los procesos de elaboración y aplicación de la justicia; proveyendo de esta manera, seguridad jurídica a los inversionistas.

- Nicaragua es el país con más potencial de crecimiento en la región, pero el que menos lo explota Los costos iniciales de inversión podrían ser elevados, pero con períodos de recuperación cortos.

Fuentes: www.irena.org, www.global-climatescope.org/en/, www,laprensa.com.ni, www.centralmericadata.com, www.elnuevodiario.com.ni, www.Radiolaprimerisima.com, 

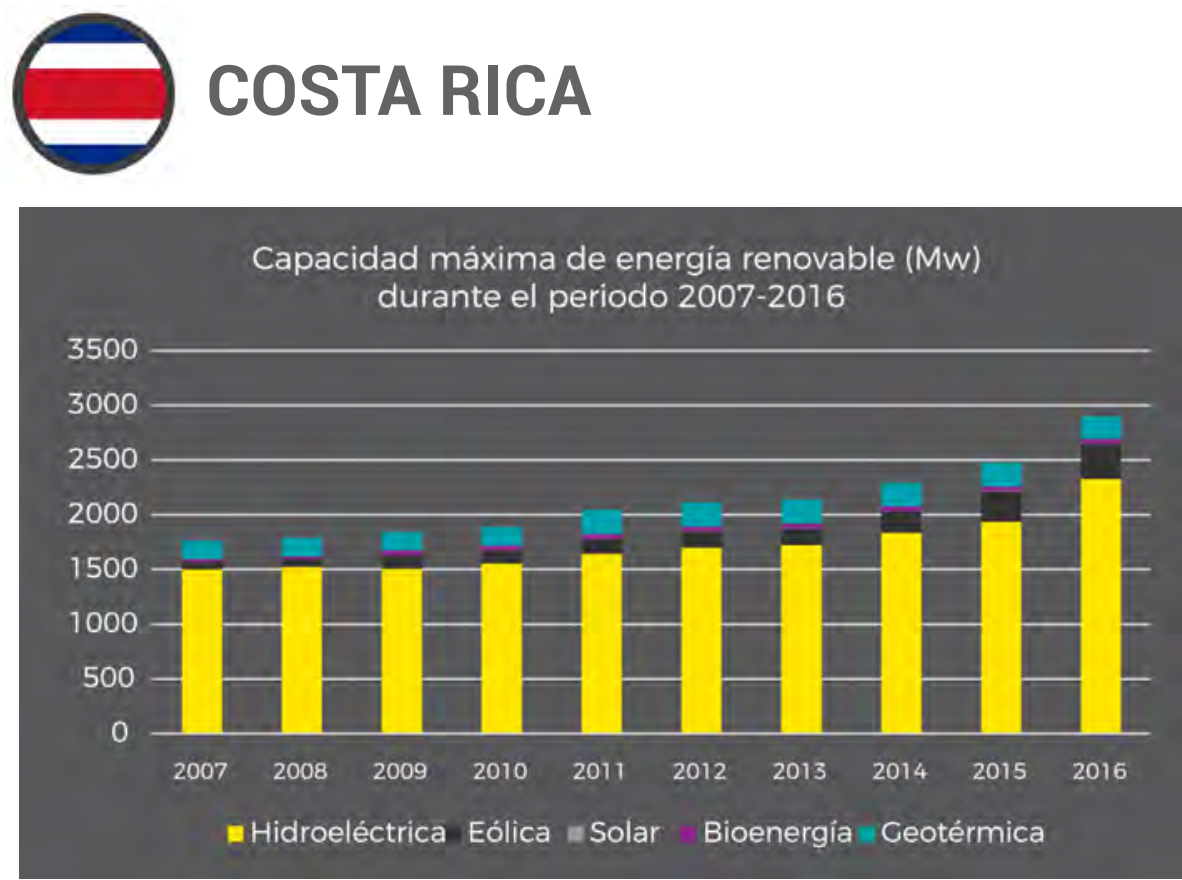

La matriz energética es altamente dependiente de la energía hidroeléctrica. La energía geotérmica ha aumentado su participación paulatinamente.

\section{A PROYECTOS FUTUROS}

\begin{tabular}{llll}
\hline Nombre & Tipo & $\begin{array}{l}\text { Inversión (US\$ } \\
\text { millones) }\end{array}$ & Generación (megavatios) \\
Borinquen I & Geotérmica & 240 & 55 \\
Las Pailas II & Geotérmica & 193,2 & 55 \\
Los Negros II & Hidrica & 120 & 28 \\
\hline
\end{tabular}

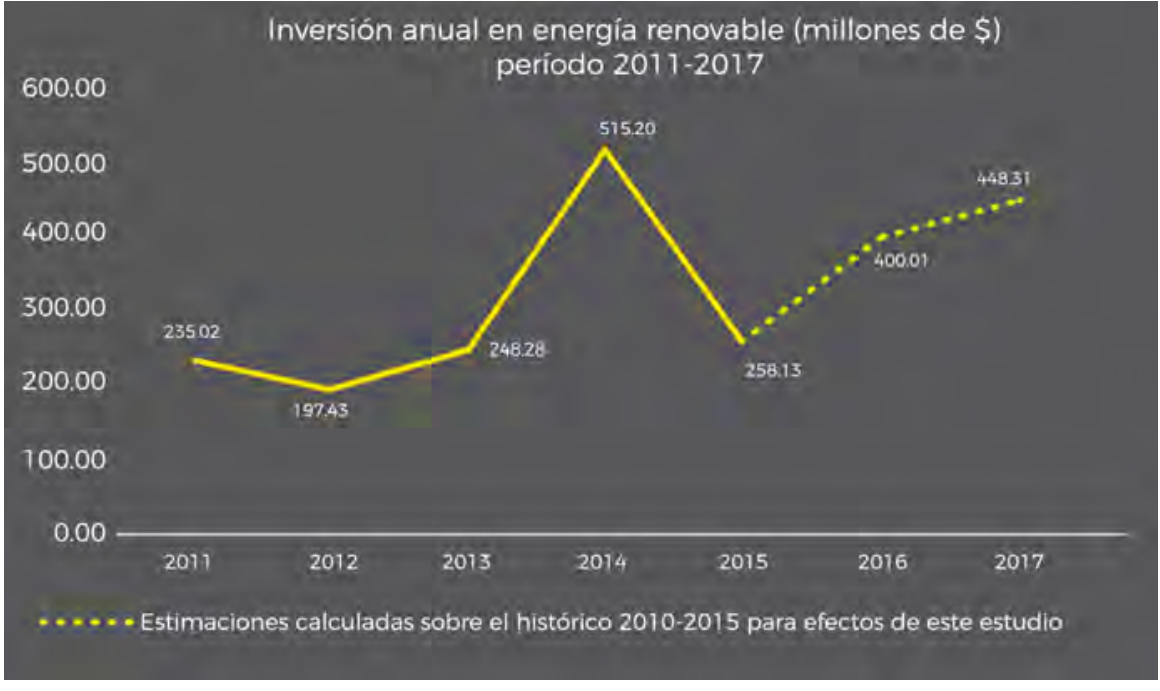

Se ha invertido más de 235 millones anuales en energía renovable excepto en el 2012, donde se invirtieron 197 millones. Se resalta que el 2014 fue el año con más inversión.

\section{$98.2 \%$}

\section{de generación de energía limpia en 2016.}

Retos país:

- Diversificar la matriz energética.

- Aumento en las cifras de inversión privada, para 2015 significó apenas el $24 \%$ del total.

- Aumento de costos en los proyectos de energía hidroeléctrica debido a que los proyectos de pequeña y mediana escala ya fueron desarrollados.

Fuentes: www.irena.org, www.global-climatescope.org/en/, www.presidencia.go.cr, www.vidayexito.net, www.grupoice.com, www.larepública. net. 


\section{$\boxplus$ PANAMÁ}

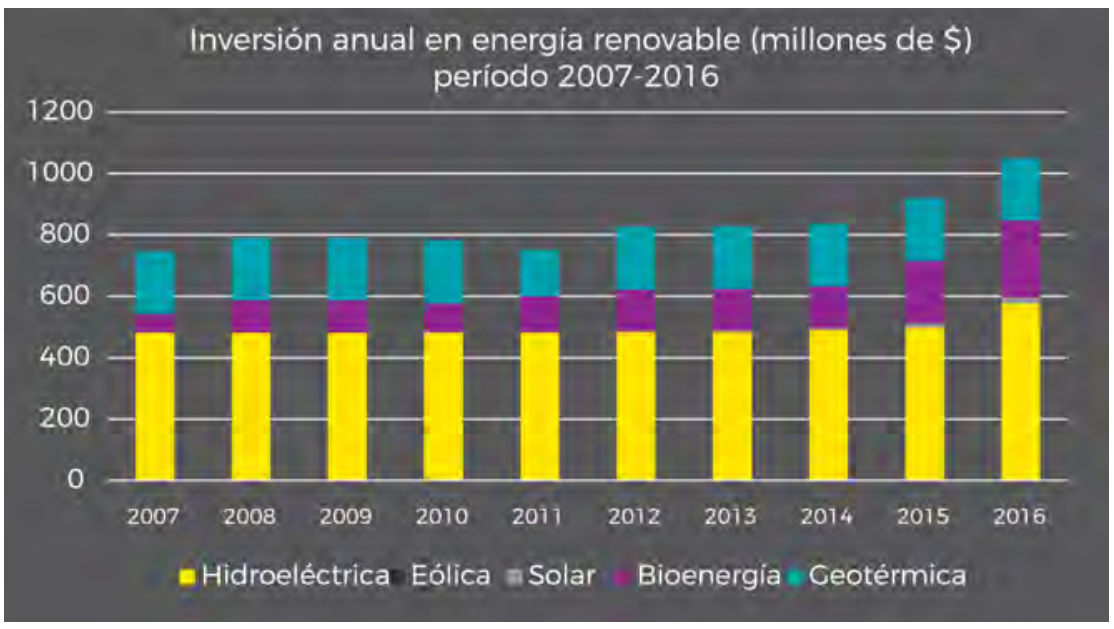

La matriz energética es altamente dependiente de la energía hidroeléctrica. La energía geotérmica ha aumentado su participación paulatinamente.

\section{PROYECTOS FUTUROS}

\begin{tabular}{|llll|}
\hline Nombre & Tipo & Inversión (US\$ millones) & Generación (megavatios) \\
Chimenea I & Eólica & N.D & 56 \\
Viento Norte & Eólica & N.D & 40 \\
La Vikinga & Eólica & 150 & 81 \\
Ikako & Solar & N.D & 10 \\
Toabre & Eólica & N.D & 225.0 \\
La Mata & Solar & N.D & 10 \\
Escudero & Eólica & N.D & 17.25 \\
\hline
\end{tabular}

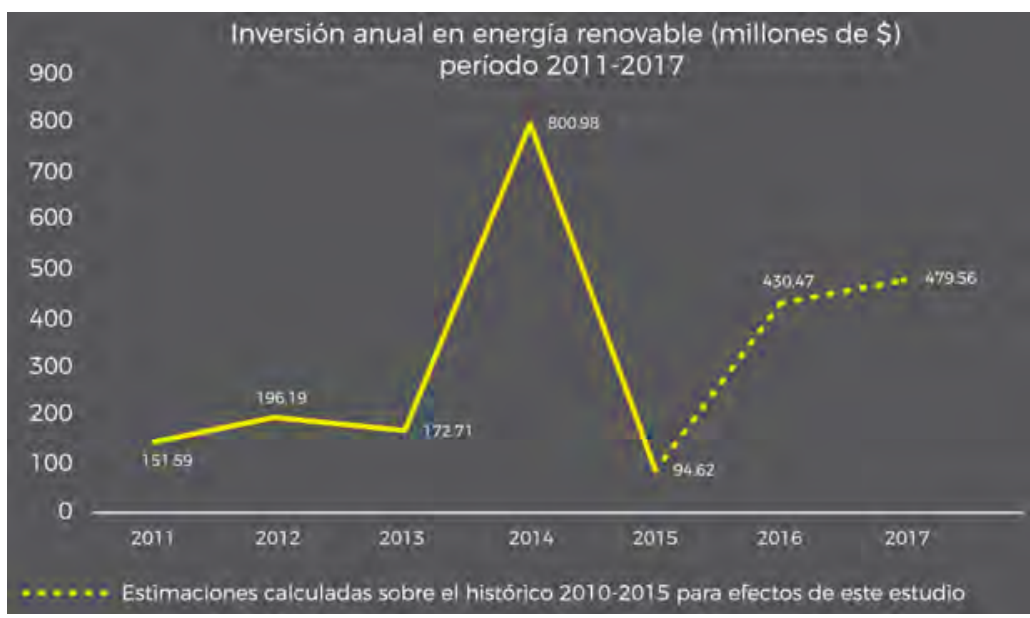

$57.1 \%$

\section{de generación de energía limpia en 2016.}

\section{Retos país:}

- Buscar fuentes de financiación.

- Diversificar la matriz energética, debido a la alta dependencia de la energía hidroeléctrica.

- Mayor uso de fuentes limpias de energía para la producción de energía eléctrica

En el año 2014 se realizó la mayor inversión en energía renovable. Hubo un importante decrecimiento para 2015 y se esperaba mayor estabilidad en la inversión para 2016 y 2017.

Fuentes: www.irena.org, www.global-climatescope.org/en/, www.americaeconomia.com, www.centralamericadata.com, www.bnamericas.com, www.evwind.com, www.prensa.com, 


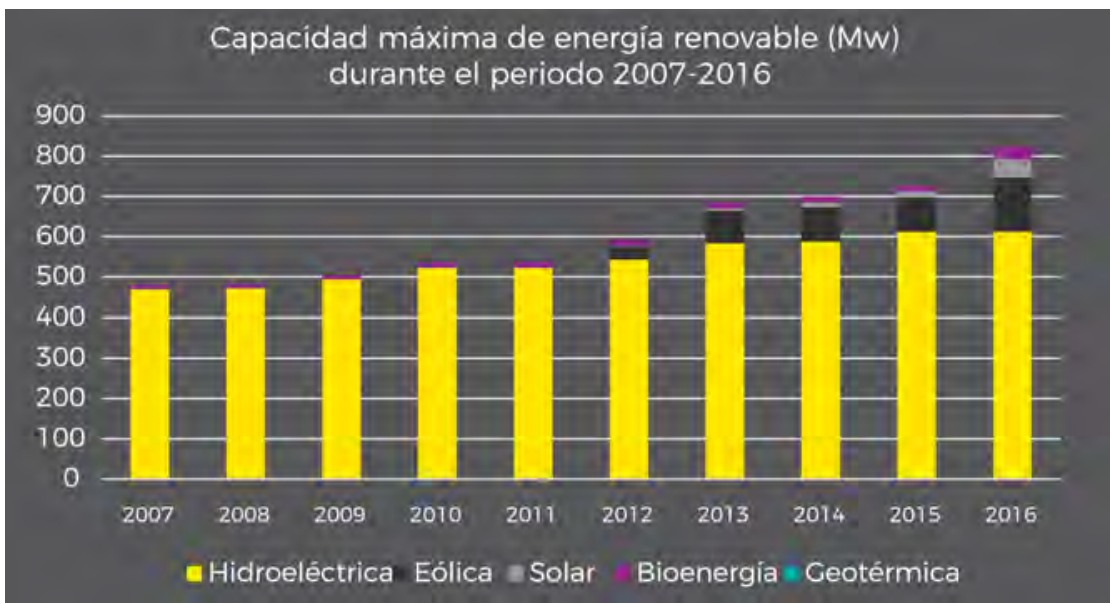

La matriz energética es altamente dependiente de la energía hidroeléctrica. La energía geotérmica ha aumentado su participación paulatinamente.

\section{A PROYECTOS FUTUROS}

\begin{tabular}{llll}
\hline Nombre & Tipo & Inversión (US\$ millones) & Generación (megavatios) \\
Emerald Solar Energy & Solar & 40 & 25 \\
Parque Eólico Matafongo & Eólica & 87.5 & 34 \\
Parque Montecristi Solar & Solar & 100 & 57.96 \\
Parque Eólico Guanillo & Eólica & 100 & 50 \\
Parque Eólico Puerto Plata & Eólica & 107.3 & 46 \\
Parque Eólico Agua Clara & Eólica & 110 & 50 \\
Parque Fotovoltaico WCG & Solar & 110 & 50
\end{tabular}

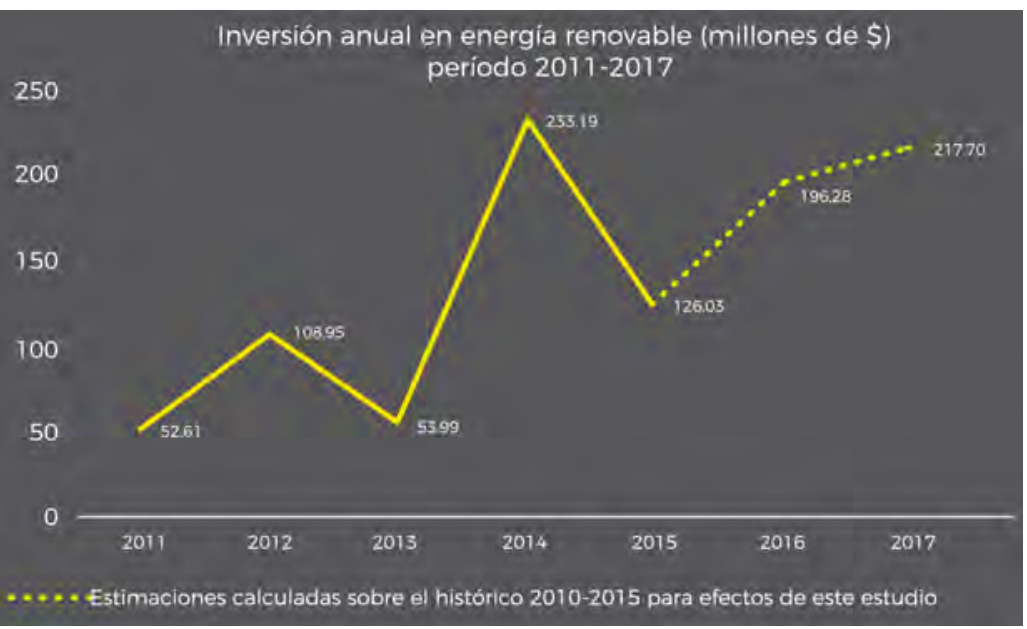

Como se puede observar en los últimos años se ha incrementado la inversión en generación de energía renovable y por lo tanto se espera cambios en la matriz energética en el mediano plazo.
$13 \%$

de generación de energía limpia en 2016.

\section{Retos país:}

- Mejorar los procesos en los que ese otorgan concesiones,

- Buscar nuevas opciones para financiar proyectos a largo plazo,

- Incentivar la inversión interna y externa en dichos proyectos proveyendo planes de recuperación de las inversiones. 


\section{TENDENCIAS EN EL SECTOR ENERGÉTICO}

\section{TECNOLOGÍAS DE ALMACENAMIENTO:}

El mayor reto del sector es solucionar la intermitencia de la generación de energía solar y eólica. A largo plazo, el bajo precio de las baterías y el aumento de su capacidad de almacenamiento podrán transformar el mercado energético. Según Thomas Christiansen, Director Asociado de EY en Stuggart, indicó que esto podría impulsar lassiguientes prácticas:

- Grid Balancing: se provee energía en el corto plazo para mantener las redes eléctricas en equilibrio. De esta manera se reduce la necesidad de invertir en redes de transmisión y distribución.

- Peak Shaving: las baterías son cargadas cuando la energía de la red es barata y se desconectan cuando es más cara. Esto reduce la demanda energética en la hora pico.

- Behind-the-meter. la energía renovable combinada con las baterías in situ permite que los usuarios puedan sacarle mayor provecho a la energía que producen. Un claro ejemplo es la utilización de la energía solar en la noche, ya que esta fue almacenada en el día.

Estos avances tecnológicos podrían conllevar a una serie de problemas, entre los cuales se destacan los siguientes:

la posible disminución de la inversión para mantener y expandir la red al reducirse la utilización de la misma y la posible baja de ingresos por parte de las empresas de servicios por la deserción de los usuarios de las redes.
Para que estas mejoras tecnológicas impacten el mercado es necesario superar las siguientes barreras:

- Barreras conceptuales: Jill Cainey, Directora del Energy Storage Network, organización que representa a la industria de almacenamiento eléctrico de Reino Unido, menciona que pensar en el almacenamiento energético es de los retos más importantes de la industria, la cual se encuentra divida entre generadores, transmisores, distribuidores, entre otros.

- Barreras regulatorias: relacionado al punto anterior, Cainey indica que en Europa es muy difícil que las empresas de servicios públicos inviertan en almacenamiento, ya las licencias son rígidas; por ejemplo, una compañía generadora de electricidad no puede almacenar energía debido a que la licencia es estrictamente de generación. Además, no existe un entendimiento claro sobre lo que se entiende por almacenamiento: ¿Es generación?, ¿Es demanda energética? ¿Quién la puede operar?, entre otros.

- Barreras técnicas: las baterías, según Cainey, son menos rentables cuando superan las cuatro horas de almacenamiento y existen en el mercado tecnologías que satisfacen mejor esta necesidad como el hidroalmacenamiento.

La tecnología está avanzando para solucionar los problemas que enfrenta la generación de energía renovable. El éxito del modelo de negocios vinculado al almacenamiento de las baterías será dictado por las decisiones que realicen los políticos y los entes reguladores. 


\section{TENDENCIAS EN EL SECTOR ENERGÉTICO}

\section{BIG DATA \& ANALYTICS}

Un estudio realizado por Green Tech Media afirma que el mercado de software que permite conocer el consumo energético de los clientes pasará de US\$300 millones a superar los US\$2b en el 2023 en los Estados Unidos. Rigler, CEO de ONZO, menciona que la instalación de medidores inteligentes y la competencia del sector eléctrico fomentan nuevas formas para analizar datos.

La compañía ONZO combina la ciencia de los datos, el análisis energético, la información brindada por los electrodomésticos y el análisis de comportamiento para conocer cómo y dónde se utiliza la energía. Esto permite que la compañía de servicios eléctricos y el cliente se beneficien de dicho conocimiento. Esta compañía trabajo dos años con SSE, proveedor de energía más grande del país, analizando datos energéticos. Los resultados fueron los siguientes:
- Se redujo el consumo eléctrico de la mayoría de los clientes.

- La mitad de los clientes redujeron un $12 \%$ el consumo eléctrico por más de dos años.

- Los usuarios más eficientes lograron reducir un $40 \%$ el uso eléctrico.

- El traslado de clientes a otro proveedor se redujo a la mitad.

A nivel mundial muchas industrias y empresas se enfocan en la administración de la energía, sin embargo nadie lo hace en los hogares. Rigler considera que esto es una oportunidad para aplicar las técnicas de eficiencia energética en el sector residencial.

\section{SISTEMA DE ALMACENAMIENTO}

En los últimos años ha aumentado la utilización de paneles solares en los techos, sin embargo la energía se desperdiciaba. Es por esta razón que Ken Munson y Dean Sanders, fundadores de Sunverge, produjeron el Sunverge Solar Integration System, el cual tiene como objetivo capturar la energía solar y utilizarla sea se considere necesario. Este sistema determina automáticamente si la energía debe ser almacenada o comprada, en caso de que la batería se encuentre descargada.

Lo anterior puede traer una serie de ventajas al consumidor y a la empresa de servicios públicos. Con respecto al primero, la factura eléctrica puede disminuir considerablemente (en algunos casos hasta $80 \%$ ) y en relación al segundo, al conocer los cambios que existen en las líneas pueden planificar dónde deben invertir los recursos.
Aunque Sunverge se dedique al a venta de software, tuvieron que diseñar el hardware para la administración de la batería, el cual realiza 900 lecturas cada 4 a 12 segundos, para obtener información de su funcionamiento, de la temperatura, entre otros factores.

La compañía ha tenido éxito puesto que en el 2010 instaló el primer sistema y para el 2016 tenían más de 1000 sistemas instalados en casas, escuelas, edificios en los Estados Unidos, Australia, Nueva Zelanda y Corea del Sur. Se puede pensar que la compañía es pequeña en comparación a las grandes compañías eléctricas, sin embargo sus creadores la consideran como una de las más grandes y con mayor experiencia debido a la novedad de dicho servicio en el mercado. 


\section{TENDENCIAS EN EL SECTOR ENERGÉTICO}

\section{ENERGÍA EÓLICA OFFSHORE}

El gobierno de Inglaterra, quién tiene el mayor mercado de energía eólica offshore decidió bajar el coste normalizado de la energía (Levelized Cost of Energy) a un terció para el 2020, sin embargo dicha meta se alcanzó en el 2016.

La disminución de los precios se debe a la duplicación de la capacidad de cada turbina, lo cual disminuye a la mitad el número de cimentaciones que se necesitan para producir la misma cantidad de energía. Otros factores que han colaborado con la reducción de los costos son la implementación de políticas públicas que apoyen el sector energético y el aumento de la competencia.
Con respecto al aumento de la competencia cabe indicar que el éxito de la energía eólica offshore ha provocado que la percepción de riesgo disminuya, provocando que los inversionistas reduzcan su retorno de inversión.

Algunos analistas señalan que los países fuera de Europa se podrían beneficiar de alguno de los factores que redujeron los costos: la innovación tecnológica, la experiencia acumulada, entre otros.

No obstante lo anterior, las cadenas de suministro local podrían tomar tiempo en desarrollar y podrían existir algunas barreras regulatorias que agregan costos. 


\section{¿CÓMO EY PUEDE APOYAR A LAS EMPRESAS QUE DESEAN INCURSIONAR EN EL ÁMBITO DE ENERGÍAS RENOVABLES?}

En la actualidad tanto empresas públicas como privadas se encuentran ante el desafío de mantener un desempeño ambiental positivo y ser rentables en el mercado. EY cuenta con amplia experiencia apoyando a empresas líderes a nivel global y de mercados emergentes como Centroamérica.

\section{Nuestra asesoría en transacciones:}

Fusiones y Adquisiciones: asesoría en procesos de inversión, asesoría en servicios de adquisición, estrategia en fusiones y adquisiciones y procesos de desincorporación carve-out.

Financiamiento de proyectos: optimización de la estructura del capital y apoyo del levantamiento del capital, asesoría en refinanciamiento, asesoría en contratación de suministros de energía (PPÁ's), asesoramiento en alianzas público-privados (asesoramiento del sector público y privado).

Estrategia e inteligencia de mercado: planeación y análisis estratégico, inteligencia de mercados (evaluación de competidores, fijación de precios en contratos de suministros, análisis regulatorio, comparativo de parámetros de valuación) y estrategia de entrada en el mercado.

Valuación: valuación comercial (valuación de inversiones, fusiones, adquisiciones y refinanciamiento, etc.), asesoría de valuación para efectos fiscales, valuación de activos intangibles (conexión a la red, opciones para expansión de parques eólicos, etc) y alojamiento de precio de compra (PPA).
Modelos financieros: modelos de negocio/valuación y asesoría en el proceso de decisiones (creando modelos para apoyo en transacciones, reestructuraciones, financiamiento de proyectos y apoyo en transacciones estratégicas como join ventures, participaciones en nuevos mercados, estrategias comerciales, etc.), asesoriamiento económico (preparación de modelos para análisis económicos), revisión de modelos de valuación.

Due dilegence financiero, fiscal y de operaciones: apoyo en procesos de desinversión carve-out, asesoría en procesos de adquisición - due dilegence, asesoría en procesos de venta - due diligence de venta, diagnostico de procesos de desinversión - éxit readiness.

Capital de Trabajo: gestión de cuentas por pagar, gestión de cartera de crédito, optimización operativa en procesos internos, optimización de liquidez.

Asesoría fiscal: optimización de estructura/carga fiscal relacionada con decisiones de inversión (estructuración fiscal nacional e internacional), asesoramiento en impuestos en transacciones de adquisición o desinversión, optimización de la estructura tributaria (IVA, impuestos de traslado de dominio, optimización en el uso de atributos fiscales existentes, etc.).

\section{Opinión}

Uno de los retos más importantes para las empresas que desean incursionar en energía renovable es la inversión inicial requerida para desarrollar los proyectos, así como poder obtener el financiamiento requerido para efectuarlos a tasas competitivas.

Desde la línea de Transaction Advisory Services, hemos brindado servicios relacionados con $M \& A$, valoración y revisión de valoración de proyectos de energía hidroeléctricos y fotovoltaicos, así como apoyo en la consecución de financiamiento para este tipo de proyectos.

Entre las tendencias globales, definitivamente la energía generada a través del método de paneles solares (fotovoltaica), es una de las más fuertes que está influenciando la región. En general todo lo que disminuya el uso de hidrocarburos y fomente las energías limpias será bienvenido. Normalmente, este tipo de proyectos están presentando aproximadamente un período de retorno de inversión de 10 a 15 años. En donde vemos que se concentra más la inversión en este tema ha sido en Panamá y Costa Rica.

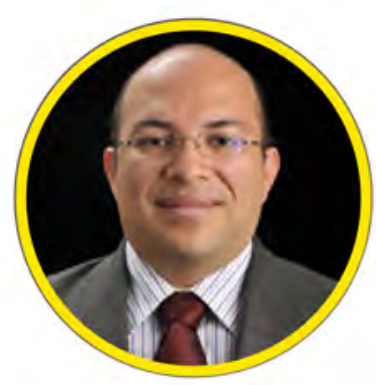

\section{Esteban Brenes}

Executive Director Transaction Advisory Services Esteban.Brenes@cr.ey.com Teléfono: 2208-9800 ext: 9975 


\section{EY | Assurance | Tax | Transactions | Consulting}

\section{Acerca de EY}

EY es un líder global en servicios de auditoría, impuestos,

transacciones y consultoría. Nuestro conocimiento, puntos de vista y servicios de calidad ayudan a generar confianza en los mercados. de capital y en las economías alrededor del mundo. Desarrollamos líderes excepcionales que trabajan en equipo para cumplir las promesas hechas a todos nuestros grupos de interés. Al hacerlo. desempeñamos un papel fundamental en la construcción de un mejor mundo de negocios para nuestra gente, nuestros clientes y nuestras comunidades.

EY se refiere a la organización global de firmas miembro conocidas como Ernst \& Young Global Limited, en la que cada una de ellas actúa como una entidad legal separada. Ernst \& Young Global Limited, compañía del Reino Unido limitada por garantía, no provee servicios a clientes. Más información en www.ev.com.

(0) 2017 E\&Y Central America Inc.

Todos los derechos reservados.

Esta publicación contiene información en forma de resumen v, por 10 tanto, su uso es solo para orientación general. No debe considerarse como sustituto de la investigación detallada o del ejercicio de un criterio profesional. Ni E\&Y Central America Inc., ni ningún otro miembro de la organización global de EY acepta responsabilidad alguna por la pérdida ocasionada a cualquier persona que actúe o deje de actuar como resultado de algún contenido en esta publicación. Sobre cualquier asunto en particular, referirse al asesor apropiado.

Los puntos de vista de terceros expuestos en la presente publicación no necesariamente son los puntos de vista de la organización globa de EY o de sus firmas integrantes. Por ende, dichos puntos de vista se deben tomar en el contexto del momento en que se expresaron.

ey.com

Centroamérica

8 Caribe

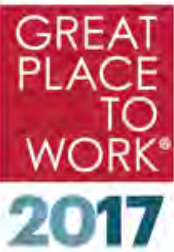

Los Mejores Lugare CA\&C

\section{E) Contacto}

Carmen Rovira

Energy Sector Leader

Partner I LATAM North

Assurance

$+(507) 2080100$

carmen:rovira@gt.ey.com

\section{Natanael Ayala}

Executive Director / Assurance and Advisory Business Services $+(503) 22487000$ natanael.ayala@sv.ey.com

\section{Gustavo Ortiz}

Director I Transaction Advisory

Services

$+(506) 22089800$

gustavo.ortiz@cr.ey.com

\section{Marcelo Nigro}

Business Development \& Com

munications

$+(506) 2208-9800$

marcelo.nigro@cr.ey.com 